\title{
Parametric estimation in photovoltaic modules using the crow search algorithm
}

\author{
Oscar Danilo Montoya ${ }^{1,2}$, Carlos Alberto Ramírez-Vanegas ${ }^{3}$, Luis Fernando Grisales-Noreña ${ }^{4}$ \\ ${ }^{1}$ Facultad de Ingeniería, Universidad Distrital Francisco José de Caldas, Bogotá D.C., Colombia \\ ${ }^{2}$ Laboratorio Inteligente de Energía, Universidad Tecnológica de Bolívar, Cartagena, Colombia \\ ${ }^{3}$ Facultad de Ciencias Básicas, Universidad Tecnológica de Pereira, Pereira, Colombia \\ ${ }^{4}$ Departamento de Electromecánica y Mecratrónica, Instituto Tecnológico Metropolitano, Medellín, Colombia
}

\begin{abstract}
Article Info
Article history:

Received Feb 1, 2021

Revised May 20, 2021

Accepted Jun 16, 2021

\section{Keywords:}

Crow search algorithm Manufacturer information Metaheuristic optimization Parametric estimation Photovoltaic modules Single-diode model

\section{ABSTRACT}

The problem of parametric estimation in photovoltaic (PV) modules considering manufacturer information is addressed in this research from the perspective of combinatorial optimization. With the data sheet provided by the PV manufacturer, a non-linear non-convex optimization problem is formulated that contains information regarding maximum power, open-circuit, and short-circuit points. To estimate the three parameters of the PV model (i.e., the ideality diode factor $(a)$ and the parallel and series resistances $\left(R_{p}\right.$ and $\left.R_{s}\right)$ ), the crow search algorithm (CSA) is employed, which is a metaheuristic optimization technique inspired by the behavior of the crows searching food deposits. The CSA allows the exploration and exploitation of the solution space through a simple evolution rule derived from the classical PSO method. Numerical simulations reveal the effectiveness and robustness of the CSA to estimate these parameters with objective function values lower than $1 \times 10^{-28}$ and processing times less than $2 \mathrm{~s}$. All the numerical simulations were developed in MATLAB 2020a and compared with the sine-cosine and vortex search algorithms recently reported in the literature.
\end{abstract}

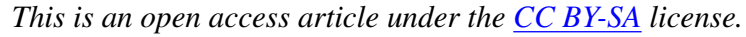

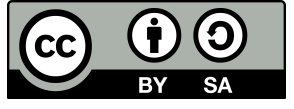

\section{Corresponding Author:}

Oscar Danilo Montoya

Facultad de Ingeniería, Proyecto Curricular de Ingeniería Eléctrica, Universidad Distrital Francisco José de Caldas

Cra 7 \# 40B-53, Bogotá D.C., Colombia

Email: odmontoyag@udistrital.edu.co

\section{INTRODUCTION}

The presence of photovoltaic (PV) sources has increased rapidly in the past two decades in low, medium and high-voltage levels, and their accelerated development has decreased their production, maintenance, and operative costs [1]-[3]. Moreover, these renewable energy resources have reduced the energy purchase costs in urban areas and greenhouse gas emissions in rural networks powered by diesel generators [4]. The integration of these PV sources into electrical grids generally requires the power electronic converters to manage their energy production to maximize the producer's profit [5]. This energy management is achieved through linear and non-linear control strategies applied to find and maintain the operation of the PV module in the maximum power point (MPP) [6]-[8]. In the literature, different models are utilized to represent the PV modules, which are composed of one, two, or three diodes. Each one of them has multiple parameters that have to be found prior to determining the panel behavior regarding current, voltage, and power outputs [2], [9]. The most accepted model to represent the PV module is the single-diode representation. In this model, we need 
to find three parameters of the PV module that are associated with the ideality diode factor and the series and parallel resistances [10]. To find these parameters, in the current literature, several optimization techniques are available that use the data sheet information provided by the panel manufacturer, where three main operative points can be highlighted: i) open-circuit point, ii) short-circuit point, and iii) MPP. These points formulate a non-linear non-convex optimization problem to determine the best combination of the model parameters to represent the complete electrical behavior of the PV module [11], [12].

In the literature, methods such as the adaptive differential evolution algorithm [5], the sine-cosine algorithm [10], the analytical method [13], the genetic algorithm [14], the cuckoo search algorithm [15], the least square method [16], the electromagnetic-like algorithm [17], and the grasshopper optimization algorithm [18], [19], among others, can be found. It should be noted that this revision demonstrates that the non-linear, non-convex nature of the parametric estimation problem makes the application of powerful optimization techniques necessary to find the optimal parameters that ensure the correct operation of the PV modules' equivalent electric circuit.

Based on this state-of-the-art revision, we propose a new optimization algorithm to estimate the electrical parameters of the PV module using the single-diode model representation. The proposed algorithm corresponds to the crow search algorithm (CSA), which has not been previously applied to this problem using the PV data sheet with the main advantage that only four parameters can be tuned. Moreover, numerical results demonstrate the values of objective functions that are lower than $1 \times 10^{-28}$, which are clearly better than the results reported in [5] and [10], where the values of objective functions were $1 \times 10^{-12}$ and $1 \times 10-15$, respectively. An additional advantage of the proposed approach is that it reaches the optimal solution in less than $2 \mathrm{~s}$ by ensuring optimal funding through the non-parametric Wilcoxon test. It is important to mention that the CSA has previously been reported in [2] to determine the parameters of the PV modules. However, the authors of [2] focus their study on an optimization model that takes into account only the power tracking error, considering variations in the temperature and irradiance inputs. It differs from our proposal since we are working directly with the manufacturer nameplate in which three operative points are considered to determine the general model of the PV system, which correspond to short-circuit point, open-circuit point, and MPP.

The remainder of this paper is organized as follows. Section 2 presents the formulation of the parametric estimation problem in PV modules considering the data sheet information provided by the manufacturer. Section 3 presents the general description of the proposed CSA. Section 4 presents the primary characteristics of the test system and the computational validation features. Section 5 shows the numerical validation of the proposed methodology and its analysis and discussion. Finally, section 6 presents the main concluding remarks derived from this research as well as some possible future works.

\section{OPTIMIZATION MODEL}

The nomenclature of the mathematical optimization model presented for the parametric estimation in PV modules using a single-diode model has been listed below:

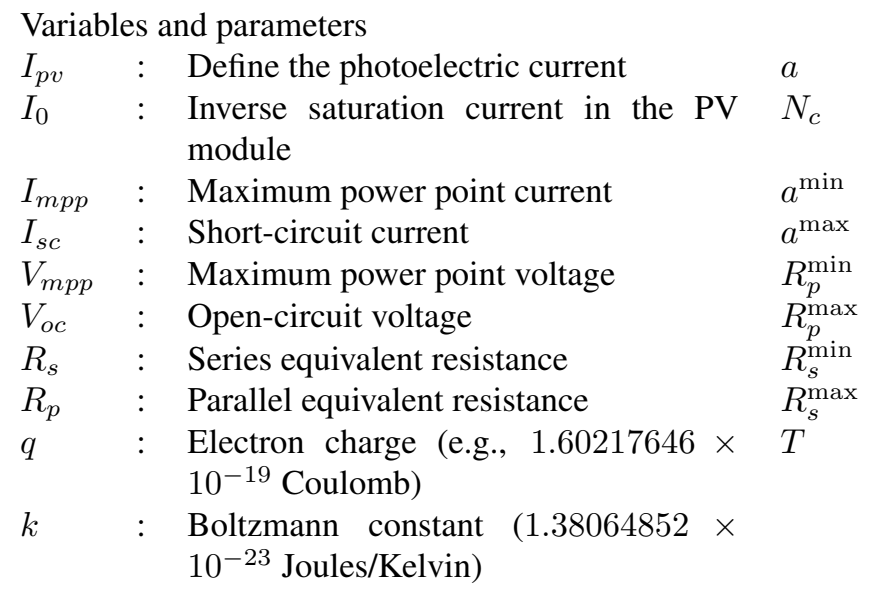

\author{
Ideality factor of the diode \\ Number of PV cells connected in series \\ $a^{\min }:$ Lower bound of the ideality diode factor \\ max : Upper bound of the ideality diode factor \\ $R_{p}^{\min }: \quad$ Lower bound of the parallel resistance \\ $R_{p}^{\max }:$ Upper bound of the parallel resistance \\ $R_{s}^{\min }:$ Lower bound of the series resistance \\ $R_{s}^{\max }:$ Upper bound of the series resistance \\ Absolute temperature in the diode union \\ $(273.15+25$ Kelvin $)$
}

The parametric estimation problem in PV modules considering $N_{c}$ cells connected in series is developed based on their ideal model using a single-diode representation [10]. The schematic modeling of the 
single-diode model, that is, the electrical circuit equivalent, has been presented in Figure 1.

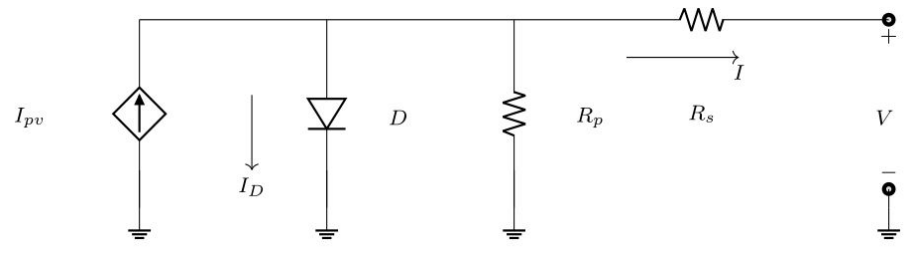

Figure 1. Equivalent circuit of a photovoltaic module

To analyze PV modules with the electrical circuit equivalent depicted in Figure 1, the exponential relation is used to define the output voltage and current as in (1) [20], [21].

$$
I=I_{p v}-I_{0}\left[\exp \left(q \frac{V+R_{s} I}{a k N_{c} T}\right)-1\right]-\frac{V+R_{s} I}{R_{p}} .
$$

With the objective to determine all the parameters of the PV module, that is, the series and parallel resistances and the diode ideality factor, the three main operative points provided by the module manufacturer have been considered. These operative points include: i) open-circuit point, ii) short-circuit point, and iii) MPP. The analysis of each one of these points has been presented below.

\subsection{Open-circuit operative point}

The open-circuit operative point of the PV module presented in Figure 1 implies that the voltage in its terminals is $V=V_{o c}$ with a null current flow through them, that is, $I=0$. With this operative condition, it is possible to obtain an expression for $I_{p v}$ from (1) as (2):

$$
I_{p v}=I_{0}\left[\exp \left(q \frac{V_{o c}}{a k N_{c} T}\right)-1\right]-\frac{V_{o c}}{R_{p}} .
$$

\subsection{Short-circuit operative point}

The second operative point provided by the PV module manufacturer corresponds to the short-circuit scenario at the terminals of the module, which implies that $I=I_{s c}$ and $V=0$. With these operative conditions, in (1) assumes in (2):

$$
I_{s c}=I_{p v}-I_{0}\left[\exp \left(q \frac{R_{s} I_{s c}}{a k N_{c} T}\right)-1\right]-\frac{R_{s} I_{s c}}{R_{p}} .
$$

Now, if we combine (2) and (3), and some algebraic manipulations are made, the following equation for the inverse saturation current is derived:

$$
I_{0}=\frac{I_{s c}+\frac{R_{s} I_{s c}}{R_{p}}+\frac{V_{o c}}{R_{p}}}{\exp \left(q \frac{V_{o c}}{a k N_{c} T}\right)-\exp \left(q \frac{R_{s} I_{s c}}{a k N_{c} T}\right)} .
$$

It should be noted that if we substitute (4) in (2), we can obtain a general representation of the PV current as (5):

$$
I_{p v}=\frac{\left(I_{s c}+\frac{R_{s} I_{s c}}{R_{p}}+\frac{V_{o c}}{R_{p}}\right)\left[\exp \left(q \frac{V_{o c}}{a k N_{c} T}\right)-1\right]}{\exp \left(q \frac{V_{o c}}{a k N_{c} T}\right)-\exp \left(q \frac{R_{s} I_{s c}}{a k N_{c} T}\right)}-\frac{V_{o c}}{R_{p}} .
$$

\subsection{Maximum power point}

In the information provided by the PV module manufacturer, an operational point named MPP is available. This point presents the information regarding the maximum possible power transferred from the panel to the system with which this is interconnected, that is, $\left(I_{m p p}, V_{m p p}\right)$. If this point is substituted in (1), a general equation for $I_{m p p}$ is found as (6):

$$
I_{m p p}=\left(\begin{array}{c}
I_{p v}-I_{0}\left[\exp \left(q \frac{V_{m p p}+R_{s} I}{a k N_{c} T}\right)-1\right] \\
-\frac{V_{m p p}+R_{s} I_{m p p}}{R_{p}} .
\end{array}\right) .
$$




\subsection{Optimization model}

Based on the information provided by the PV module manufacturer, that is, the three aforementioned operative points, it is possible to formulate an optimization model that allows the estimation of the parameters of the electrical equivalent circuit, that is, series and parallel resistances and the ideality diode factor using a single-objective formulation that minimizes the mean square error between the manufacturer data and the calculated values. The general structure of the objective function assumes the following form:

$$
\min z=E_{o c}^{2}+E_{s c}^{2}+E_{m p p}^{2}
$$

where

$$
\begin{aligned}
E_{o c} & =I_{0}\left[\exp \left(q \frac{V_{o c}}{a k N_{c} T}\right)-1\right]-\frac{V}{R_{p}}-I_{p v}, \\
E_{s c} & =I_{p v}-I_{0}\left[\exp \left(q \frac{R_{s} I_{s c}}{a k N_{c} T}\right)-1\right]-\frac{R_{s} I_{s c}}{R_{p}}-I_{s c}, \\
E_{m p p} & =\left(\begin{array}{c}
I_{p v}-I_{0}\left[\begin{array}{c}
\left.\exp \left(q \frac{V_{m p p}+R_{s} I}{a k N_{c} T}\right)-1\right] \\
-\frac{V_{m p p}+R_{s} I_{m p p}}{R_{p}}
\end{array}\right)-I_{m p p} .
\end{array}\right.
\end{aligned}
$$

It should be noted that to find the value of the objective function defined in (7), it is necessary to know the values of the parameters $a, R_{s}$, and $R_{p}$ (decision variables) in conjunction with the simultaneous solution of the (4) and (5) for the inverse saturation and the PV current. To complete the optimization model for parametric estimation in PV modules considering manufacturer data, we assign the lower and upper bounds for the decision variables as in (11) [10]:

$$
\begin{gathered}
a^{\min } \leq a \leq a^{\max }, R_{p}^{\min } \leq R_{p} \leq R_{p}^{\max } \\
R_{s}^{\min } \leq R_{s} \leq R_{s}^{\max }
\end{gathered}
$$

It should be observed that the proposed optimization model defined from (7) to (11) added with the equality constraints (4) and (5) corresponds to a continuous non-linear non-convex optimization problem. It implies that multiple solutions for the parameters $a, R_{s}$, and $R_{p}$ can exist with the same numerical performance, that is, multimodal optimization behavior [22]. Due to this reason, to reach an adequate solution with minimal computational effort, this paper proposes the application of the methaeuristic optimization technique known as CSA [23], [24], which has not been previously reported in the literature to address the parametric estimation problem in PV modules. This is the main contribution of this research based on its excellent numerical performance. In the next section, we will present the CSA and its application of the studied problem.

\section{CROW SEARCH ALGORITHM}

The CSA is a recently developed combinatorial optimization technique to solve continuous non-linear complex and non-convex optimization problems with multiple constraints [25], [26]. This technique belongs to the family of the bio-inspired optimization derived from the conventional particle swarm optimization (PSO) methodology. It is commonly known that crows observe other birds to know where they hide their food in order to steal it once the owner leaves. In the case a crow commits thievery, this crow will take additional precautions such as moving hiding places to reduce the possibility of being a future victim [27]. In fact, they use their own experience of having been a thief to forecast the behavior of possible thieves and can determine the safest course to protect their caches from being pilfered. The main characteristics of the crows are: i) they live in flocks; ii) they can memorize the position of their hiding places; iii) they can follow each other to commit thievery; and iv) they can protect their caches from being pilfered by a probability. In this paper, we present the mathematical adaptation of this behavior to solve complex optimization problems as originally proposed in [23]. The primary steps in the implementation of the CSA have been discussed below.

\subsection{Initialization of the problem and selection of the adjustable parameters}

The optimization problem is defined, that is, the optimization model (7) to (11) is added with the equality constraints (4) and (5). Then, the adjustable parameters of the CSA are selected, that is, the flock size $n$, the maximum number of iterations $t_{\max }$, the flight length $f_{l}$, and the awareness probability $A_{p}$. It should be 
noted that the selection of these parameters is heuristic and depends on the knowledge that the programmer has about the optimization problem under study. These are typically selected using multiple simulations to identify the best trade-off between response quality and processing times.

\subsection{Initial position and memory of the crows}

In a $d$-dimensional space, all the $n$ crows are initially positioned as the members of the flock. It is worth mentioning that each crow represents a feasible solution of the optimization problem that is composed by $d$ decision variables.

$$
\text { Crows }=\left[\begin{array}{cccc}
x_{11} & x_{12} & \cdots & x_{1 d} \\
x_{21} & x_{22} & \cdots & x_{2 d} \\
\vdots & \vdots & \ddots & \vdots \\
x_{n 1} & x_{n 2} & \cdots & x_{n d}
\end{array}\right]
$$

It should be noted that the generation of each component of the crow $i$ associated with the variable $j$ takes the following form:

$$
x_{i j}=x_{j}^{\min }+r\left(x_{j}^{\max }-x_{j}^{\min }\right), \forall\left\{\begin{array}{l}
i=1,2, \ldots, n \\
j=1,2, \ldots, d
\end{array}\right\}
$$

where, $r$ is a random number between 0 and 1 generated with a normal distribution.

In the beginning of the search process, the memory of each crow is initialized. However, in the initial iteration, the crows have no search experience. Then, it is assumed that they have hidden their foods at their initial positions.

$$
\text { Memory }=\left[\begin{array}{cccc}
m_{11} & m_{12} & \cdots & m_{1 d} \\
m_{21} & m_{22} & \cdots & m_{2 d} \\
\vdots & \vdots & \ddots & \vdots \\
m_{n 1} & m_{n 2} & \cdots & m_{n d}
\end{array}\right]
$$

\subsection{Fitness function evaluation}

As a conventional metaheuristic optimization algorithm, the CSA works with a fitness function instead of the original objective function of the problem to deal with infeasibilities in the solution space [28]. However, in the case of the parametric estimation of PV modules, due to the structure of the optimization model, it is possible to directly evaluate the objective function for each crow.

\subsection{Generation of the new position for each crow}

The generation of new positions for the crows in the search space proceeds as follows: consider that the crow $i$ wants to pass to a new position. For this goal, this crow randomly selects one of the flock crows (e.g., crow $k$ ) to follow in order to discover the position where they have hidden their food (i.e., $m_{k}$ ). Following this procedure, the new position of the crow $i$ is obtained as (16):

$$
x_{i}^{t+1}=\left\{\begin{array}{cc}
x_{i}^{t}+r_{i} f_{l}^{i, t}\left(m_{k}^{t}-x_{i}^{t}\right), & r_{k} \geq A_{p}^{k, t} \\
\text { a random position (see (13)), } & \text { otherwise }
\end{array}\right.
$$

where $r_{j}$ is a random number with uniform distribution between 0 and 1 , and $A_{p}^{k, t}$ denotes the awareness probability of crow $k$ at iteration $t$. It should be observed that this procedure is repeated for all the crows. It is worth mentioning that each crow generated with (15) is revised if the lower and upper bounds of the decision variables are fulfilled. In the case that the crow $i$ violates these bounds, in (13) is used to correct it.

\subsection{Evaluation of the objective function and updating of the memories}

For each one of the positions of the crows, the objective function is evaluated, which is used to update their memories as (16):

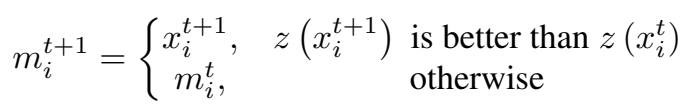

It is observed that if the fitness function value of the new position of the crow $i$ is better than the fitness function value of the memorized position, the crow updates its memory by the new position. "Better" in the case of parametric estimation in PV modules implies "lower". 


\subsection{Check termination criterion}

The steps defined in subsections 3.4. and 3.5. are repeated until the maximum number of iterations is reached (i.e., $t_{\max }$ ). When the termination criterion is met, the best position of the memory in terms of the objective function value (i.e., the minimum value) is reported as the solution of the optimization problem.

\subsection{Implementation of the CSA}

Algorithm 1 presents the general pseudo-code that defines the steps necessary to implement the CSA to solve the problem of parametric estimation in PV modules [23].

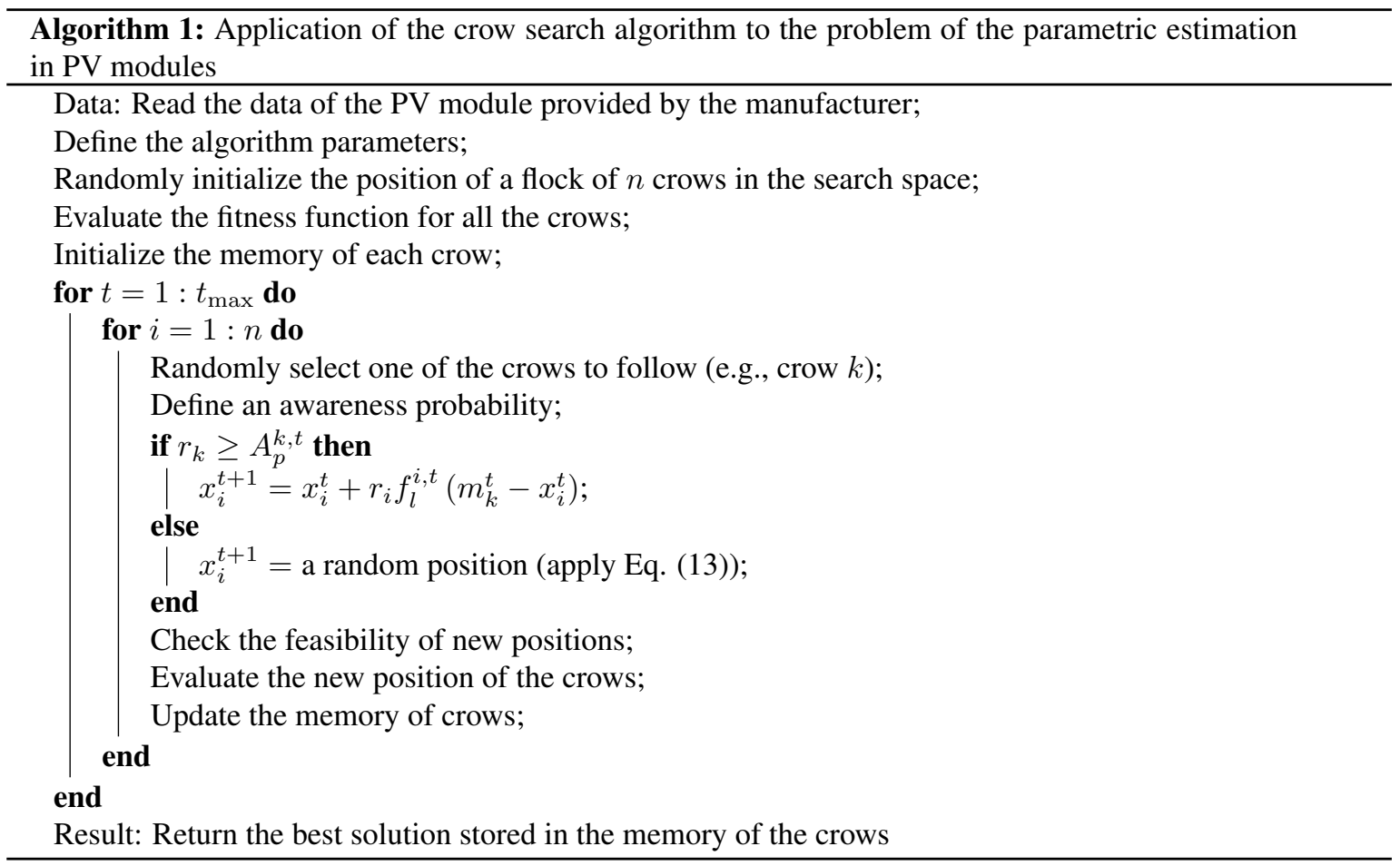

\section{TEST SYSTEM AND COMPUTATIONAL VALIDATION}

The implementation of the proposed CSA to the problem of the parametric estimation in PV modules represented with its single-diode model has considered the information provided by the manufacturer of the Kyocera KC200GT [10] as shown in Table 1, in the software MATLAB 2020a using a desk computer INTEL(R) Core(TM) $i 5-3550$ 3.5-GHz, 8 GB of RAM version 64-bit with Microsoft Windows 7 Professional.

To validate the effectiveness of the CSA to find a high-quality solution to the problem of the parametric estimation in PV modules, a population of 20 individuals was considered, that is, $n=20$, with 100000 consecutive iterations; an awareness probability fixed as 0.75 , and a variable fly length defined by the following rule $f_{l}=2$ rand $*\left(1-t / t_{\max }\right)$. Moreover, the lower and upper bounds for the decision variables are $0.5 \leq a \leq 2,0.001 \leq R_{p} \leq 1$, and $50 \leq R_{s} \leq 200$, which have been taken from [10].

Table 1. Kyocera KC200GT manufacturer information (Taken from [5])

\begin{tabular}{ccc}
\hline Parameter & Symbol & Value \\
\hline Open-circuit voltage & $V_{o c}$ & $32.900 \mathrm{~V}$ \\
Temperature coefficient for $V_{o c}$ & $K_{V_{o c}}$ & $-0.123 \mathrm{~V} /{ }^{\circ} \mathrm{C}$ \\
Short-circuit current & $I_{s c}$ & $8.210 \mathrm{~A}$ \\
Temperature coefficient for $I_{s c}$ & $K_{I_{s c}}$ & $3.180 \times 10^{-3} \mathrm{~A} /{ }^{\circ} \mathrm{C}$ \\
Voltage on the MPP & $V_{m p p}$ & $26.300 \mathrm{~V}$ \\
Number of cell in series & $N_{c}$ & 54 \\
Current on the MPP & $I_{m p p}$ & $7.610 \mathrm{~A}$ \\
\hline
\end{tabular}




\section{NUMERICAL RESULTS}

The application of the CSA to the problem of parametric estimation in PV modules produced the results reported in Table 2, where the best 10 solutions are presented after 100 consecutive evaluations. From results in Table 2, we can observe that: i) the value of the objective function related with the mean square error (see (7)) that evaluates the error regarding the open-circuit point, short-circuit point, and MPP provided by the manufacturer of the PV module and the calculated values using the single-diode model are lower (i.e., better) than $1 \times 10^{-29}$, which can be considered null for any practical implementation. In this context, as mentioned in [5], all the parameters represent optimal solutions, moreover, these improve the conclusion reported in [10] wherein values lower than $x^{-15}$ were considered optimal; ii) the solutions in the range from 3 to 8 present the same objective function value, that is, $7.8886 \times 10^{-31}$, which confirm the multimodal nature of the problem of the parametric estimation in PV modules since there are different combinations of the decision variables that have the same numerical performance; iii) the electrical parameter that presents more variations along the optimal solutions is the parallel resistance since the minimum value reached for this parameters is found in the solution 10 with a value of $55.0001 \Omega$ and the maximum value is found in the solution 5 with a value of $188.3342 \Omega$, that is, a difference superior than $120 \Omega$ between both solutions; and iv) the average processing times reported by the CSA to find the numerical results reported in Table 2 was about $1.80 \mathrm{~s}$ with a standard deviation of $0.20 \mathrm{~s}$, which demonstrates the efficiency of the CSA to find the global optimal solution.

In Figure 2 is presented the $V-I$ curve of the PV module for each one of the ten solutions reached by the CSA and presented in Table 2. These curves were obtained making a sweep in the voltage variable from 0 to $V_{o c}$ in steps of $0.10 \mathrm{~V}$ by solving the (1) for all the combinations of $a, R_{s}$ and $R_{p}$ parameters of the single-diode model of the PV module presented in Figure 1. From the numerical results presented in Figure 1 , it can be noted that the points $P_{1}, P_{2}$, and $P_{3}$ correspond to the open-circuit point, MPP, and short-circuit operative points, which confirms that the information provided by the PV module manufacturer is sufficient to estimate with minimum errors the complete behavior of the panel in all its operative range; moreover, when the Wilcoxon test was applied for ten independent samples, each one of them with 10 optimal solutions, a mean value for $p$ of about $p$ of 0.5486 with a value of $h=0$ was obtained; this implies that the null hypothesis of the Wilcoxon test is confirmed, and therefore, the analyzed samples present the same median with a significance level of $100 \%$, which demonstrates that the CSA has the ability to find the global optimal solution at each evaluations with 100 consecutive search through the solution space.

Table 2. Ten best results reached by the CSA

\begin{tabular}{ccccc}
\hline $\mathrm{N}_{\mathbf{o}}$ & $a$ & $R_{s}(\Omega)$ & $R_{p}(\Omega)$ & $f_{f}$ \\
\hline 1 & 0.65018 & 0.3938 & 56.8600 & 0 \\
2 & 0.57158 & 0.5199 & 81.3492 & 0 \\
3 & 0.59211 & 0.4996 & 76.4901 & $7.8886 \times 10^{-31}$ \\
4 & 0.64397 & 0.5071 & 113.2315 & $7.8886 \times 10^{-31}$ \\
5 & 0.50549 & 0.6038 & 188.3341 & $7.8886 \times 10^{-31}$ \\
6 & 0.51566 & 0.5436 & 74.2629 & $7.8886 \times 10^{-31}$ \\
7 & 0.58256 & 0.5137 & 81.6568 & $7.8886 \times 10^{-31}$ \\
8 & 0.60683 & 0.5451 & 159.1443 & $7.8886 \times 10^{-31}$ \\
9 & 0.67940 & 0.3646 & 55.8052 & $2.2877 \times 10^{-29}$ \\
10 & 0.67623 & 0.3605 & 55.0001 & $2.5243 \times 10^{-29}$ \\
\hline
\end{tabular}

\subsection{Comparison with combinatorial methods}

In this section, we present the comparative results among the proposed CSA and different combinatorial methods that solve optimization problems in the continuous domain. These methods are: vortex search algorithm [12], sine-cosine algorithm [10], PSO [29], and genetic algorithm [30]. For each one of these comparative methods, 100 consecutive evaluations were made to obtain the best 10 results reported in Table 3 .

From results listed in Table 3 it is possible to observe that:

- The CSA and the VSA optimization approaches present the best numerical performance with respect to the objective function values lower than $\times 10^{-28}$ and $\times 10^{-25}$, respectively.

- The solutions found by the SCA and the CGA methods can also be considered optimal, since the objective function is in practical terms null. However, we can mention that the SCA presents a better numerical performance when compared with the CGA as was demonstrated in [10], since the best objective function is $9.7192 \times 10^{-28}$ for the SCA and $3.2207 \times 10^{-12}$ for the CGA, which implies a difference higher than $1 \times 10^{-5}$ between them. 
- The list of different solutions reported in 3 confirms the non-linear non-convex nature of the problem of the parametric estimation in PV systems, since the CGA and the SCA are stuck in local optimums, while the PSO, the VSA, and the proposed CSA present a better numerical performance reaching high-quality objective function values.

To complement the analysis among the CSA and the comparative metaheuristic methods, the parameters for the optimal solutions are listed in Table 4 with label number 1 presented in Table 3.

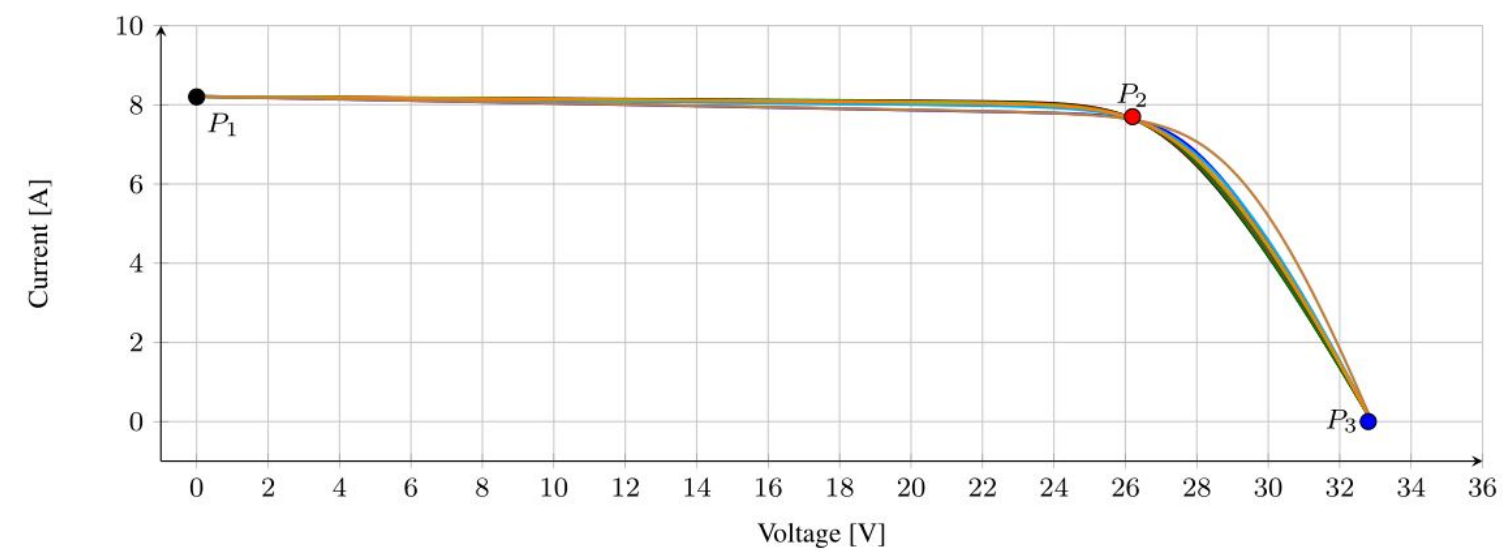

Figure 2. $V-I$ curve obtained by solving (1) for the parameters presented in Table 2

Table 3. Ten best solutions reported by each comparative method

\begin{tabular}{cccccc}
\hline $\mathrm{N}_{\mathbf{0}}$ & CSA & VSA & SCA & PSO & CGA \\
\hline 1 & 0 & 0 & $9.7192 \times 10^{-18}$ & $2.5243 \times 10^{-29}$ & $3.2207 \times 10^{-12}$ \\
2 & 0 & 0 & $1.0823 \times 10^{-17}$ & $1.0097 \times 10^{-28}$ & $5.1049 \times 10^{-11}$ \\
3 & $7.8886 \times 10^{-31}$ & 0 & $1.3799 \times 10^{-16}$ & $1.6155 \times 10^{-27}$ & $2.3014 \times 10^{-10}$ \\
4 & $7.8886 \times 10^{-31}$ & 0 & $1.9364 \times 10^{-16}$ & $2.5243 \times 10^{-27}$ & $2.7546 \times 10^{-10}$ \\
5 & $7.8886 \times 10^{-31}$ & $7.8886 \times 10^{-29}$ & $3.5309 \times 10^{-16}$ & $6.0647 \times 10^{-27}$ & $3.9197 \times 10^{-10}$ \\
6 & $7.8886 \times 10^{-31}$ & $1.9248 \times 10^{-28}$ & $5.2122 \times 10^{-16}$ & $2.9181 \times 10^{-26}$ & $5.1134 \times 10^{-10}$ \\
7 & $7.8886 \times 10^{-31}$ & $1.3067 \times 10^{-26}$ & $5.4738 \times 10^{-16}$ & $3.5498 \times 10^{-26}$ & $5.2351 \times 10^{-10}$ \\
8 & $7.8886 \times 10^{-31}$ & $1.3354 \times 10^{-26}$ & $8.4393 \times 10^{-16}$ & $5.0553 \times 10^{-26}$ & $6.0393 \times 10^{-10}$ \\
9 & $2.2877 \times 10^{-29}$ & $2.9614 \times 10^{-26}$ & $9.3922 \times 10^{-16}$ & $7.6361 \times 10^{-26}$ & $6.2646 \times 10^{-10}$ \\
10 & $2.5243 \times 10^{-29}$ & $4.8872 \times 10^{-26}$ & $1.5123 \times 10^{-16}$ & $1.4485 \times 10^{-25}$ & $6.8811 \times 10^{-10}$ \\
\hline
\end{tabular}

Table 4. Optimal solutions reported by the proposed and comparative methods

\begin{tabular}{cccc}
\hline Method & $a$ & $R_{s}(\Omega)$ & $R_{p}(\Omega)$ \\
\hline CSA & 0.650181877806710 & 0.393806884684579 & 56.8600309871423 \\
VSA & 0.502572297672421 & 0.505917172241395 & 57.6920080257133 \\
PSO & 0.681740933460645 & 0.508343460036893 & 170.884395321487 \\
SCA & 0.917140758347724 & 0.146864384999908 & 52.6718647012995 \\
CGA & 0.985461664181760 & 0.234102605899478 & 70.5159926098178 \\
\hline
\end{tabular}

\section{CONCLUSION}

In this research, the CSA was implemented to find the optimal parameter combination to represent PV modules with its single-diode model. Numerical results demonstrated that this algorithm finds solutions with values lower than $1 \times 10^{-28}$ regarding the objective function value after 100 consecutive evaluations, which were better in comparison with the classical metaheuristic methods used to solve this problem; these methods were the VSA, SCA, PSO, and CGA respectively. The first 10 solutions reached by the CSA confirm that the problem of the parametric estimation in PV modules is a multimodal non-linear optimization problem with different combinations of the decision variables that present the same numerical performance. Regarding the processing times, the proposed CSA takes about $1.80 \mathrm{~s}$ to find the optimal solution of the studied problem with the main advantage being that based on the Wilcoxon test, after 100 consecutive evaluations, the possibility 
of finding the global optimum is ensured. Moreover, the CSA is easily implementable in any programming language with only 4 parameters to be tuned. In the future, it will be possible to develop the following research works: i) to extend the proposed CSA to the parametric estimation in induction motors and distribution transformers that are modeled with non-linear non-convex optimization models and ii) to apply the proposed optimization model to the estimation of parameters in PV modules considering real measures of voltages and currents including variable weather conditions.

\section{ACKNOWLEDGMENTS}

This work was supported in part by the Centro de Investigación and Desarrollo Científico de la Universidad Distrital Francisco José de Caldas under grant 1643-12-2020 associated with the project: "Desarrollo de una metodología de optimización para la gestión óptima de recursos energéticos distribuidos en redes de distribución de energía eléctrica." and in part by the Dirección de Investigaciones de la Universidad Tecnológica de Bolívar under grant PS2020002 associated with the project: "Ubicación óptima de bancos de capacitores de paso fijo en redes eléctricas de distribución para reducción de costos and pérdidas de energía: Aplicación de métodos exactos and metaheurísticos."

\section{REFERENCES}

[1] M. S. Cengiz and M. S. Mamis, "Price-efficiency relationship for photovoltaic systems on a global basis," International Journal of Photoenergy, vol. 2015, pp. 1-12, 2015, doi: 10.1155/2015/256101.

[2] A. Omar, H. M. Hasanien, M. A. Elgendy, and M. A. L. Badr, "Identification of the photovoltaic model parameters using the crow search algorithm," The Journal of Engineering, vol. 2017, no. 13, pp. 1570-1575, Jan. 2017.

[3] A. Paz-Rodriguez, J. F. Castro-Ordo nez, O. D. Montoya, and D. A. Giral-Ramırez, "Optimal integration of photovoltaic sources in distribution networks for daily energy losses minimization using the vortex search algorithm," Applied Sciences, vol. 11, no. 10, May. 2021, Art. No. 4418, doi: 10.3390/app11104418.

[4] O. D. Montoya, L. F. Grisales-Nore na, W. Gil-Gonzalez, G. Alcala, and Q. Hernandez-Escobedo, "Optimal location and sizing of PV sources in DC networks for minimizing greenhouse emissions in diesel generators," Symmetry, vol. 12, no. 2, Feb. 2020, Art. No. 322, doi: 10.3390/sym12020322.

[5] P. P. Biswas, P. Suganthan, G. Wu, and G. A. Amaratunga, "Parameter estimation of solar cells using datasheet information with the application of an adaptive differential evolution algorithm," Renewable Energy, vol. 132, pp. 425-438, 2019, doi: 10.1016/j.renene.2018.07.152.

[6] D. G. Montoya, C. A. R. Paja, and R. Giral, "Maximum power point tracking of photovoltaic systems based on the sliding mode control of the module admittance," Electr. Power Syst. Res., vol. 136, pp. 125-134, Jul. 2016, doi: 10.1016/j.epsr.2016.02.001.

[7] M. Al-Soeidat, D. D.-C. Lu, and J. Zhu, "An analog BJT-tuned maximum power point tracking technique for PV systems," IEEE Transactions on Circuits and Systems II: Express Briefs, vol. 66, no. 4, pp. 637-641, Apr. 2019, doi: 10.1109/TCSII.2018.2865804.

[8] M. Arjun, V. V. Ramana, R. Viswadev, and B. Venkatesaperumal, "An iterative analytical solution for calculating maximum power point in photovoltaic systems under partial shading conditions," IEEE Transactions on Circuits and Systems II: Express Briefs, vol. 66, no. 6, pp. 973-977, Jun. 2019, doi: 10.1109/TCSII.2018.2867088.

[9] A. R. Jordehi, "Parameter estimation of solar photovoltaic (PV) cells: A review," Renewable Sustainable Energy Rev., vol. 61, pp. 354-371, Aug. 2016, doi: 10.1016/j.rser.2016.03.049.

[10] O. D. Montoya, W. Gil-Gonzalez, and L. F. Grisales-Nore na, "Sine-cosine algorithm for parameters' estimation in solar cells using datasheet information,” Journal of Physics: Conference Series, vol. 1671, Oct. 2020, Art. No. 012008.

[11] U. Jadli, P. Thakur, and R. D. Shukla, "A new parameter estimation method of solar photovoltaic," IEEE Journal of Photovoltaics, vol. 8, no. 1, pp. 239-247, 2018, doi: 10.1109/JPHOTOV.2017.2767602.

[12] W. J. Gil-Gonzalez, O. D. Montoya, and J. M. Lopez-Lezama, "Vortex search algorithm applied to the parametric estimation in pv cells considering manufacturer datasheet information," IEEE Latin America Transactions, vol. 19, no. 9, pp. 1581-1589, Sep. 2021, doi: 10.1109/TLA.2021.9468612.

[13] A. Elkholy and A. A. El-Ela, "Optimal parameters estimation and modelling of photovoltaic modules using analytical method," Heliyon, vol. 5, no. 7, p. e02137, Jul. 2019, doi: 10.1016/j.heliyon.2019.e02137.

[14] M. A. Awadallah and B. Venkatesh, "Estimation of PV module parameters from datasheet information using optimization techniques," in 2015 IEEE International Conference on Industrial Technology (ICIT), Mar. 2015, doi: 10.1109/ICIT.2015.7125507.

[15] J. Ma, T. O. Ting, K. L. Man, N. Zhang, S.-U. Guan, and P. W. H. Wong, "Parameter estimation of photovoltaic models via cuckoo search," J. Appl. Math., vol. 2013, pp. 1-8, 2013, doi: 10.1155/2013/362619.

[16] N. Maouhoub, "Photovoltaic module parameter estimation using an analytical approach and least squares method," J. Comput. Electron., vol. 17, no. 2, pp. 784-790, Jan. 2018, doi: 10.1007/s10825-017-1121-5.

[17] H. M. Ridha, C. Gomes, and H. Hizam, "Estimation of photovoltaic module model's parameters using an improved electromagneticlike algorithm," Neural Computing and Applications, vol. 32, no. 16, pp. 12627-12642, Jan. 2020, doi: 10.1007/s00521-020-04714$\mathrm{z}$.

[18] O. S. Elazab, H. M. Hasanien, I. Alsaidan, A. Y. Abdelaziz, and S. M. Muyeen, "Parameter estimation of three diode photovoltaic model using grasshopper optimization algorithm ," Energies, vol. 13, no. 2, Jan. 2020, Art. No. 497, doi: 10.3390/en13020497. 
[19] J. Montano, A. F. Tobon, J. P. Villegas, and M. Durango, "Grasshopper optimization algorithm for parameter estimation of photovoltaic modules based on the single diode model," International Journal of Energy and Environmental Engineering, vol. 11, no. 3, pp. 367-375, Feb. 2020, doi: 10.1007/s40095-020-00342-4.

[20] A. K. Abdulrazzaq, G. Bognar, and B. Plesz, "Accurate method for PV solar cells and modules parameters extraction using i-v curves," Journal of King Saud University-Engineering Sciences, Jul. 2020, doi: 10.1016/j.jksues.2020.07.008.

[21] R. Ndegwa, J. Simiyu, E. Ayieta, and N. Odero, "A fast and accurate analytical method for parameter determination of a photovoltaic system based on manufacturer's data," Journal of Renewable Energy, vol. 2020, pp. 1-18, May. 2020, doi: 10.1155/2020/7580279.

[22] R. Chelouah and P. Siarry, "Continuous genetic algorithm designed for the global optimization of multimodal functions," Journal of Heuristics, vol. 6, no. 2, pp. 191-213, 2000, doi: 10.1023/A:1009626110229.

[23] A. Askarzadeh, "A novel metaheuristic method for solving constrained engineering optimization prob- lems: Crow search algorithm," Computers and Structures, vol. 169, pp. 1-12, Jun. 2016.

[24] Y. Meraihi, A. B. Gabis, A. Ramdane-Cherif, and D. Acheli, "A comprehensive survey of Crow Search Algorithm and its applications," Artificial Intelligence Review, Sep. 2020.

[25] B. Zolghadr-Asli, O. Bozorg-Haddad, and X. Chu, "Crow search algorithm (CSA)," in Advanced Optimization by Nature-Inspired Algorithms, Springer Singapore, Jul. 2017, pp. 143-149, doi: 10.1007/978-981-10-5221-7-14.

[26] A. A. E. Ela, R. A. El-Sehiemy, A. Shaheen, and A. Shalaby, "Application of the crow search algorithm for economic environmental dispatch," in 2017 Nineteenth International Middle East Power Systems Conference (MEPCON), Dec. 2017, doi: 10.1109/MEPCON.2017.8301166.

[27] S. Shekhawat and A. Saxena, "Development and applications of an intelligent crow search algorithm based on opposition based learning," ISA Transactions, vol. 99, pp. 210-230, Apr. 2020, doi: 10.1016/j.isatra.2019.09.004.

[28] O. D. Montoya, W. Gil-Gonzalez, and L. F. Grisales-Norena, "Vortex search algorithm for optimal power flow analysis in DC resistive networks with CPLs," IEEE Transactions on Circuits and Systems II: Express Briefs, vol. 67, no. 8, pp. 1439-1443, Aug. 2020, doi: 10.1109/TCSII.2019.2938530.

[29] V. Khanna, B. K. Das, D. Bisht, Vandana, and P. K. Singh, "Estimation of photovoltaic cells model parameters using particle swarm optimization," in Physics of Semiconductor Devices, Springer International Publishing, 2014, pp. 391-394, doi: 10.1007/978-3-319-03002-9-98.

[30] Y. Zhang, S. Lyden, B. L. de la Barra, and M. Haque, "A genetic algorithm approach to parameter estimation for PV modules," in 2016 IEEE Power and Energy Society General Meeting (PESGM), Jul. 2016, doi: 10.1109/PESGM.2016.7741781. 